\title{
Closed-Loop Modulation of a Notch-Filter Stimulation Strategy for Tremor Management with a Neuroprosthesis
}

\author{
J.A. Gallego ${ }^{1}$, E. Rocon ${ }^{1}$, J.M. Belda-Lois ${ }^{2}$ and J.L. Pons ${ }^{1}$ \\ ${ }^{1}$ Bioengineering Group, Spanish National Research Council (CSIC), Madrid, Spain \\ 2 Instituto de Biomecánica de Valencia, Universitat Politècnica de València, Valencia, Spain
}

\begin{abstract}
Tremor is the most common movement disorder, and one of the major causes of functional disability. In spite of the existence of various treatments, tremor is not managed effectively in a large number of patients, which make it a major cause of loss of quality of life. Here we present a novel strategy for tremor suppression through neurostimulation that replicates an adaptive notch-filter at the frequency of the tremor. The controller, which adapts the neurostimulation to the ongoing amplitude, frequency and phase of the tremor, is implemented in a wearable neuroprosthesis for tremor management. Experimental results in one patient with severe essential tremor illustrate the interest of the approach.
\end{abstract}

Keywords - Tremor, Neurostimulation, Essential tremor, Neuroprosthesis, Electromechanical delay

\section{INTRODUCTION}

Tremor is the most common movement disorder, and its prevalence is expected to increase in the next decades [1]. It appears due to a number of etiologically different disorders [2], none of which is fully understood [3]. Drugs constitute the standard treatment for the various types of tremor, and refractory patients - normally with severe symptoms-may undergo neurosurgery. In spite of the existence of these various treatments, a large proportion of patients exhibit significant disability when performing their daily chores $[4,5]$. This motivates the research on novel forms to manage tremor.

Among the alternatives for tremor management that are currently under development, external devices that apply selective forces to attenuate the tremor have attracted great interest. These systems span devices fixed to a external frame and wearable orthoses, which are likely to provide the biggest functional benefit (see [6] for a brief review). Neuroprostheses (NPs) may be regarded as a special type of wearable orthoses, in which actuators are replaced by neural interfaces that stimulate the affected muscles. In [7], the authors proposed the first design of a table-mounted NP for tremor suppression, which provided a significant improvement [8] of the major types of tremor. In spite of the successful results it attained, a number of aspects should be improved to facilitate its translation into a product that improves the patients' quality of life, mainly: $i$ ) the NP should ideally be wearable system, and ii) the NP should adapt the stimulation it delivers to the ongoing characteristics of the tremor (mainly its severity). Therefore, in this work we present a novel controller for a tremor suppression NP that expands the originally proposed in [7] by implementing real-time adaptation to tremor amplitude, frequency and phase. The paper presents its concept and implementation, and provides experimental evidence in a essential tremor patient that demonstrate its potential. The strategy was implemented in a NP published elsewhere [9]. Further, we report a study of the electromechanical delay (EMD) of forearm muscles for this type of applications.

\section{TREMOR SUPPRESSION STRATEGY}

The tremor suppression strategy here presented relied on the same principle than that presented in [7]. There, the authors implemented a controller that, theoretically, stimulated a pair of antagonist muscles in counterphase to the ongoing tremor bursts. This resembled a notch filter at the selected frequency, which was selected in accordance to that expected for the different types of tremor [2,7]. However, in [7], the assumed frequency was not corrected during the experiments, which might be problematic given that the characteristics of tremor vary depending on the conditions (as the authors mentioned in the report of the experimental validation [8]). A conceptually identical strategy, but with frequency adaptation was implemented and successfully validated in a recent work [10]. However, the authors of [10] developed a controller that delivered a constant stimulation level, which may have a number of drawbacks, e.g. it may facilitate the onset of muscle fatigue, it may cause that a current density higher than needed to compensate for the ongoing tremor is injected (possibly generating discomfort), and, importantly, it may impede the performance of concurrent voluntary movements.

Therefore, we developed a new notch-filter tremor suppression strategy, in which the stimulation delivered was adapted to the ongoing amplitude, frequency and phase of the tremor. Furthermore, it implemented a mechanism to com- 
pensate for the effects of the electromechanical delay (EMD, in this case understood as the time period between the delivery of neurostimulation and movement onset), and the possibility of producing brief periods of co-contraction when the direction of rotation switches due to the tremor oscillations. In more detail, the controller (summarized in Fig. 1) was implemented as follows. First, the raw motion, recorded with a pair of solid-state gyroscopes, was separated into concomitant voluntary movement and tremor, using a two-stage adaptive algorithm [11]. Next, to obtain accurate information about which muscle needed to be stimulated, the tremor obtained from the solid-state gyroscopes was differentiated, to compute the angular acceleration. Given that tremor is a rhythmical oscillatory movement, the angular acceleration has a phase of $180^{\circ}$ relative to the angular displacement, which immediately provided the controller with information about the muscle in the antagonist pair that needed to be actuated. The estimated tremor also served to correct for the estimated tremor frequency and phase if a large discrepancy was detected (see Fig. 1). Next, the electromechanical delay was applied to the estimated tremor phase (see the right part of Fig. 1), which in turn served to choose whether stimulation needed to be delivered in co-contraction or in counterphase (see the switch in Fig. 1). The stimulation amplitude was computed using a proportional integral (PI) algorithm, and a saturation was applied to it, for comfort and safety reasons. Controller gains and the saturation values were identified during calibration. The last part of the controller (the blue rectangle in Fig. 1) was implemented independently for each muscle in the antagonist pair.

\section{Methods}

\section{A. Assessment of the Electromechanical Delay}

We quantified the EMD in forearm muscles in order to investigate: $i$ ) the possible impact of this phenomenon on the performance of the NP, and ii) whether this parameter needed to be identified for each patient, and, in the case that this was not required, obtain a general estimate. Hence, we performed a experiment on 6 young volunteers ( 3 male and 3 female, age 28-31 years), who gave informed consent to participate.

The protocol aimed to quantify the EMD defined as the interval between stimulation onset and movement onset, as detected with the gyroscopes implemented in the NP [9]. Such definition was employed because the final goal was to integrate the EMD estimate in the controller of the NP. Our experimental design addressed the influence of inter- and intra-subject variability on the EMD, the latter understood as changes in EMD due to the stimulation with different ampli- tude. To this end, we applied 15 1-s stimulation bursts (separated by a 2-s pause) to the flexor carpi ulnaris and the extensor carpi radialis while the hand was supported against gravity, and fully supinated or pronated, respectively. The 15 stimulation bursts were applied at 3 different amplitudes (5 each), defined as low (L), medium (M) and high (H). These values were visually chosen after electrode setup. Stimulation frequency and pulse width were constant, and set to $30 \mathrm{~Hz}$ and $250 \mu$ s respectively. Trial order was randomized, and the experiment designed using an optimal algorithm [12]. Hand rotation was measured with a pair of solid state gyroscopes (Technaid S.L., Madrid, Spain) placed in differential configuration [11]. Neurostimulation was delivered with a multichannel monopolar stimulator that injected charge compensated pulses (UNA Systems, Belgrade, Serbia); the common electrode was placed at the dorsal side of the wrist. The data were stored for posterior analysis. Movement onset was determined manually based on the inspection of gyroscope data. The effect of stimulation amplitude and stimulation site (i.e. muscle) on each subject's data was assessed with a one-way ANOVA with repeated measures. Differences among subjects were also assessed with a one-way ANOVA with repeated measures. All results are reported as mean $\pm \mathrm{SD}$.

\section{B. Pilot Testing of the Tremor Suppression Strategy}

We present results for one patient with essential tremor (female) with severe postural and kinetic tremor. She was recruited from a outpatient clinic, informed beforehand, and gave written informed consent to participate. The ethical committee at Universidad Politécnica de Valencia, Valencia, Spain, approved the experimental protocol, and warranted its accordance to the declaration of Helsinki.

The experimental protocol replicated that presented in [9]. Briefly, the patient was asked to perform 12 repetitions of a task that triggered her tremor, which in this case was a postural task. The repetitions were split into two types of 30-s trials (referred to as ST and NO) which order was randomized. Each trial consisted of two 15-s sub-periods. In the ST trials, neurostimulation was delivered in the second sub-period, while in the NO trials neurostimulation was never delivered. The goal of this design was to avoid the influence of inter- and intra-trial tremor variations in the results. Details on the implementation of the NP are given in [9]. As mentioned above, the gains of the controller were identified manually at the beginning of the session: the proportional term was obtained as the best linear fit to the movement data obtained after applying a series of 5 1-s stimulation bursts, with increasing amplitude; the experimental setup replicated that employed to quantity the EMD. The integral gain was initially chosen 


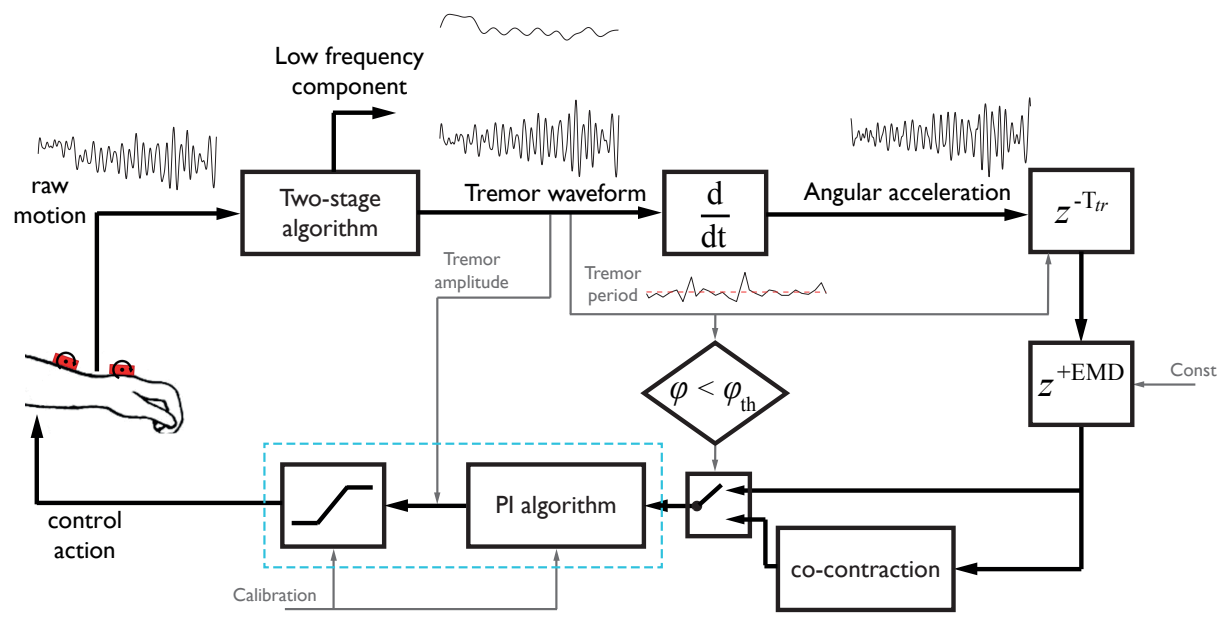

Fig. 1: Block diagram of the proposed tremor suppression strategy.

as $1 / 2$ the proportional gain. The saturation values were obtained as the maximum amplitude to elicit a movement with amplitude larger than that observed for the tremor, and perceived as comfortable by the patient. The EMD was set to 20 $\mathrm{ms}$ (according to the results presented below); the antagonist pair was co-contracted for a time period equivalent to $30^{\circ}$.

Tremor attenuation was computed as the ratio of the integral of the power spectral density (PSD) of the tremor in the second sub-period of the trial to the same variable calculated in the first sub-period $\left(R_{\mathrm{att}}\right)[13,9]$, and reported as percentage. The pooled values of $R_{\text {att }}$ for the ST and NO trials were compared using a Mann-Whitney test, to assess whether the control strategy attained a significant tremor attenuation.

\section{RESULTS}

\section{A. Electromechanical Delay}

Fig. 2 shows a representative response of the response evoked by the stimulation of the extensor carpi radialis. Similar data were obtained for the flexor carpi ulnaris. The plot shows how the magnitude of the response increased as a higher current amplitude was injected to the muscle, and that, for the same stimulation amplitude, differences among repetitions were negligible. The delay observed for different stimulation amplitudes was very similar (average $19 \pm 4 \mathrm{~ms}$ ).

The pooled EMD data for the extensor carpi radialis ( $18 \pm$ $8 \mathrm{~ms})$ and flexor carpi radialis $(22 \pm 12 \mathrm{~ms})$ did not present large discrepancies, and, for each subject, was statistically independent of the muscle $(P=0.137)$. Stimulation amplitude did not affect significantly the EMD either $(P=0.743$ and $P=$ 0.541 for the extensor carpi radials and the flexor carpi ulnaris respectively). Moreover, although we found a significant dif-

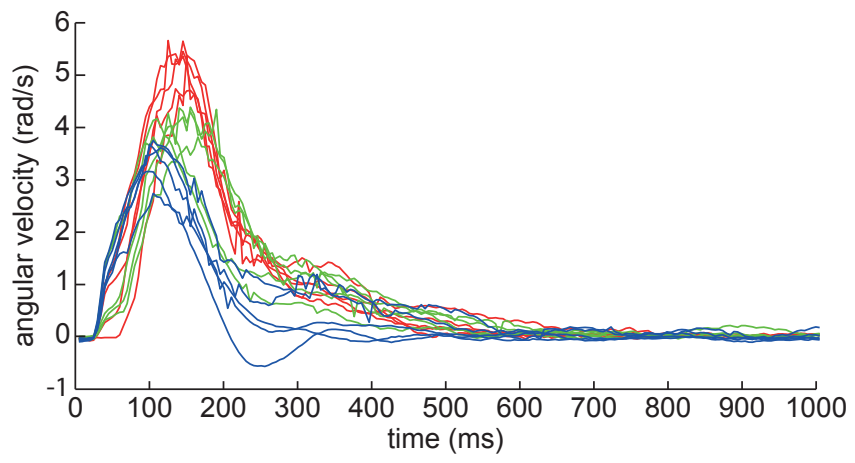

Fig. 2: Response evoked by the stimulation of the extensor carpi radialis with $\mathrm{L}$ (blue), $\mathrm{M}$ (green) and $\mathrm{H}$ (red) current amplitudes, in one subject.

ference among subjects in the EMD for the same muscle $(P$ $=0.005$ and $P<0.001$ for the extensor carpi radials and the flexor carpi ulnaris respectively) the maximum discrepancies were $9 \mathrm{~ms}$ for the extensor carpi radialis, and $16 \mathrm{~ms}$ for the flexor carpi radialis ( $3 \mathrm{~ms}$ in the latter if we remove subject 6 from the analysis, who could be considered as an outlier).

\section{B. Tremor Suppression Strategy}

Fig. 3 shows a representative example of the performance of the controller, in which we observe that the control strategy proposed attained a fast and large reduction of the tremor $\left(R_{\text {att }}\right.$ $=11.55 \%)$. The average reduction of tremor in all the ST trials was $R_{\text {att }}=16.77 \pm 8.33 \%$, ranging from 10.26 to 29.38 $\%$. This tremor reduction was significantly larger $(P=0.002)$ than that measured in the NO trials (mean $R_{\text {att }}=162.33 \pm$ $150.51 \%$, ranging from 88.18 to $463.73 \%$ ), which indicates that the NP was the cause of the observed tremor attenuation. 


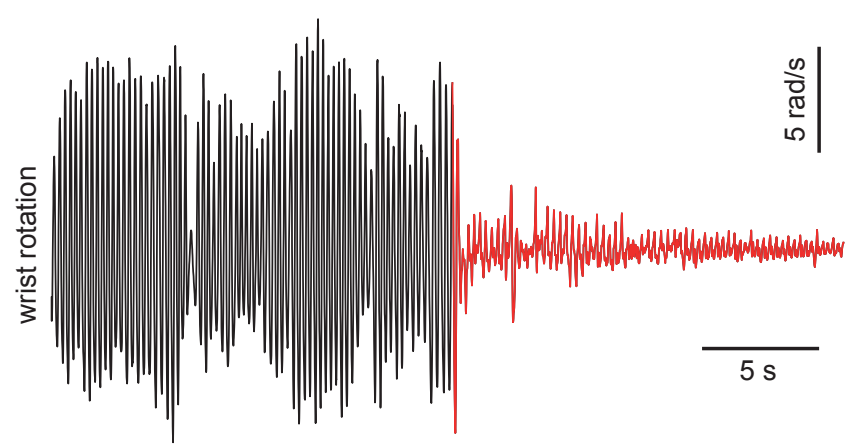

Fig. 3: Representative example of the performance of the controller, showing a large tremor attenuation. The two sub-periods of the trial are color coded (without stimulation in black, with stimulation in red).

\section{Discussion}

This paper presented a new strategy for neuroprosthetic tremor management. The strategy relied on the traditional principle for tremor suppression through transcutaneous neurostimulation, but adapted, for the first time, the electrical charge delivered to the ongoing amplitude, frequency and phase of the tremor, which is expected to translate immediately in better functional outcome. Moreover, the proposed control strategy inherently compensated for the EMD, and implemented brief, adjustable co-contraction periods, which, in our opinion, could provide an important improvement of the controller. Results in one patient with severe tremor proved the feasibility and interest of the approach.

We have first reported a study to assess the EMD in forearm muscles, since we expected that due to the rationale of the controller, and the inherent relatively high frequency of some tremors (ET may be up to $10 \mathrm{~Hz}$ [2]), it could have a large influence on the performance of the controller. Our results showed that, for the same subject, the muscle and the stimulation amplitude did not cause a significant change in the EMD. Furthermore, although we obtained a statistically significant difference among subjects, such difference was very small, and given that the controller of the NP runs at 200 $\mathrm{Hz}$, it may be neglected in the current context. We thus assumed that, for our application, the EMD may be considered to be constant, and equal to $\sim 20 \mathrm{~ms}$. This value is in agreement with the literature in the sense that the delay is shorter than for volitional contractions [14], although it cannot be directly compared given the different fashion in which it was obtained $[14,15]$. As a matter of fact, much more precise measurements of EMD, e.g. using percutaneous stimulation combined with high frequency ultrasound recordings, found significant differences when the current density was changed [15]. Therefore, the results here presented are valid for appli- cations such NPs for functional compensation of movement disorders, albeit they might not be completely generalizable.

The tremor suppression strategy provided systematic reduction of tremor amplitude, and interestingly, with a low stimulation amplitude once the tremor severity was very mild. This demonstrates the interest of adapting the amount of current injected to the ongoing severity of the tremor. Functional trials with a representative cohort of patients are needed to further demonstrate the interest of the approach.

\section{ACKNOWLEDGMENT}

This work has been partly supported by the EU Commission [NeuroTREMOR, grant number EU-FP7-2011-287739].

\section{REFERENCES}

1. Bach J.-P., Ziegler U., Deuschl G., et al . Projected numbers of people with movement disorders in the years 2030 and 2050. Mov Disord. 2011;26:2286-2290.

2. Deuschl G., Bain P., Brin M.. Consensus statement of the Movement Disorder Society on Tremor. Ad Hoc Scientific Committee. Mov Disord. 1998;13 Suppl 3:2-23.

3. Elble R.J.. Tremor: clinical features, pathophysiology, and treatment Neurologic Clinics. 2009;27:679-695.

4. Hariz G-M., Forsgren L.. Activities of daily living and quality of life in persons with newly diagnosed Parkinson's disease according to subtype of disease, and in comparison to healthy controls. Acta Neurol Scand. 2011;123:20-27.

5. Louis E. D., Barnes L., Albert S. M., et al. Correlates of functional disability in essential tremor. Mov Disord. 2001;16:914-920.

6. Gallego J. A., Rocon E., Belda-Lois J. M., et al . Design and validation of a neuroprosthesis for the treatment of upper limb tremor in Conf Proc IEEE Eng Med Biol Soc 2013 in press.

7. Prochazka A., Elek J., Javidan M.. Attenuation of pathological tremors by functional electrical stimulation. I: Method. Ann Biomed Eng. 1992;20:205-224.

8. Javidan M., Elek J., Prochazka A.. Attenuation of pathological tremors by functional electrical stimulation. II: Clinical evaluation. Ann Biomed Eng. 1992;20:225-236.

9. Gallego J. A., Rocon E., Belda-Lois J. M., Pons J. L.. A neuroprosthesis for tremor management through the control of muscle co-contraction. J Neuroeng Rehabil. 2013;10:36.

10. Popovic-Maneski Lana, Jorgovanovic Nikola, Ilic Vojin, et al. Electrical stimulation for the suppression of pathological tremor. Med Biol Eng Comput. 2011;49:1187-1193.

11. Gallego J. A., Rocon E., Roa J. O., et al . Real-Time Estimation of Pathological Tremor Parameters from Gyroscope Data Sensors. 2010;10:2129-2149.

12. Fedorov T. T.. Theory of optimal experiments. Academic Press 1972.

13. Rocon E., Belda-Lois J. M., Ruiz A. F., et al . Design and Validation of a Rehabilitation Robotic Exoskeleton for Tremor Assessment and Suppression IEEE Tans Neural Syst Rehab Eng. 2007;15:367-378.

14. Hopkins J.T., Feland J. B., Hunter I.. A comparison of voluntary and involuntary measures of electromechanical delay. Int J Neurosci. 2007;117:597-604.

15. Lacourpaille L., Nordez A., Hug F.. Influence of stimulus intensity on electromechanical delay and its mechanisms. J Electromyogr Kinesiol. 2013;23:51-55. 
Author: J.A. Gallego

Institute: CAR - CSIC

Street: Ctra. Campo Real, km. 02, La Poveda

City: Arganda del Rey, Madrid

Country: Spain

Email: ja.gallego@csic.es 